\title{
Factors associated with Staphylococcus aureus nasal carriage and molecular characteristics among the general population at a Medical College Campus in Guangzhou, South China
}

B. J. Chen ${ }^{1+}$, X. Y. Xie ${ }^{1+}$, L. J. Ni ${ }^{1 \dagger}$, X. L. Dai ${ }^{1}$, Y. Lu ${ }^{2}, X . Q$ Q. Wu ${ }^{1}$, H. Y. Li ${ }^{1}$, Y. D. Yao ${ }^{3 *}$ and S. Y. Huang ${ }^{1 *}$

\begin{abstract}
Background: The nasal cavity is the main colonization site of Staphylococcus aureus (S. aureus) in human body. Nasal carriage may be a strong risk factor for some serious infection. There was still limited information about the nasal carriage for S. aureus in south China.

Methods: Sought to determine the prevalence and molecular characteristics of S. aureus nasal carriage, 295 volunteers residing on a medicine campus were investigated and sampled the nasal cavity swab. Selected S. aureus isolates were carried through molecular analysis, including pulsed-field gel electrophoresis (PFGE), multilocus sequence analysis, staphylococcal cassette chromosome mec (SCCmec) and virulence gene detection.

Results: A total of 73 S. aureus isolates were recovered from separate subjects (24.7\%, 73/295), with one methicillinresistant S. aureus (MRSA) isolate $(0.3 \%, 1 / 295)$. Among the 73 isolates, 71 isolates were successfully grouped into 13 pulsotypes by PFGE analysis, with profiles $A$ and $L$ the most prevalent; 12 sequence types (STs) were found among the 23 isolates which had similar drug resistant spectrum. ST59, ST188 and ST1 were the most prevalent, accounting for 17.4, 13.0 and $13.0 \%$ of all isolates, respectively. The MRSA isolate presented ST8-SCCmec III. 56.5\% of isolates carried both the staphylococcal enterotoxin A (sea) and enterotoxin B (seb) genes. 83.6\% of the S. aureus isolates were resistant to penicillin, all isolates were susceptible to quinupristin/dalfopristin, levofloxacin, teicoplanin and vancomycin. The most common risk factors for $S$. aureus carriage were being male, age $\leq 30$ years, and nasal cavity cleaning habits.

Conclusions: Colonization by S. aureus was greater among male and young age (20-30 years) students and those with irregularity nasal cleaning. The $S$. aureus isolates selected were revealed into various sequence types and pulsotypes, indicating molecular heterogeneity among $S$. aureus isolates from the populations in the medical college in Guangzhou.
\end{abstract}

Keywords: Staphylococcus aureus, Nasal carriage, Antimicrobial susceptibility, Medical population, Molecular characteristics

*Correspondence: yaoyand@mail.sysu.edu.cn; hsongyin@126.com ${ }^{\dagger}$ B. J. Chen, X. Y. Xie and L. J. Ni contributed equally to this work ${ }^{1}$ Department of Laboratory, Guangdong Provincial Key Laboratory of Malignant Tumor Epigenetics and Gene Regulation, Sun Yat-Sen Memorial Hospital, Sun Yat-Sen University, Guangzhou 510120, China

${ }^{3}$ Breast Tumor Center, Sun Yat-Sen Memorial Hospital, Sun Yat-Sen University, Guangzhou 510120, Guangdong, China

Full list of author information is available at the end of the article provided you give appropriate credit to the original author(s) and the source, provide a link to the Creative Commons license, and indicate if changes were made. The Creative Commons Public Domain Dedication waiver (http://creativecommons.org/ publicdomain/zero/1.0/) applies to the data made available in this article, unless otherwise stated. 


\section{Background}

Staphylococcus aureus (S. aureus) can cause nosocomial and community-acquired infections in humans. As a medically pathogen, colonization is a strong risk factor and serious threat to human health. Multiple sites of the human body can be the ecological niche of $S$. aureus, but the main colonization site is the anterior nares [1]. The rates of infection in persistent carriers are higher than others [2]. Besides young age and being male, the main factors to colonize strains were the usage of antibiotics, chronic disease and hospitalization [3-5]. S. aureus could spread by contacting with a colonized individual [5]. That may be the reason why individuals without any healthcare-associated risk factors $[6,7]$ could have led to an increased awareness of community-associated methicillin-resistant $S$. aureus (CA-MRSA). Besides, the $S$. aureus that leads to an invasive infection were in distinguishable from carriage isolates previously isolated from the anterior nares by pulsed-field gel electrophoresis [8, 9] in a patient who gave pulmonary infection. The key to understand the transmission potential of S. aureus is to unravel the risk factors for carriage of $S$. aureus. Molecular typing of $S$. aureus is helpful for supporting infection control measures, investigating suspected outbreaks, and preventing nosocomial transmission $[10,11]$. Nasal carriage rates were different among races. Moreover, the frequency with which $S$. aureus can be detected in the nose if human individuals, was shown to differ among people of different histocompatibility antigen types (HLA) [12, 13]. It still remains unclear whether carriage rates and risk factors among the Chinese populations that typically lived under crowded conditions are in the same range. And date on $S$. aureus nasal carriage among populations that typically gathered under crowded conditions in Guangzhou is very limited. The medical students need to carry through many experiments which relate to microorganism and go on a field trip to a hospital during their undergraduate career, so they are more likely to expose to the $S$. aureus. Therefore, we sought to determine the prevalence and risk factors of $S$. aureus nasal carriage from students, teachers, retirees among community residents at the campus of Zhongshan School of Medicine, Sun Yat-sen University (SYSU), Guangzhou, South China.

\section{Methods}

\section{Population and study design}

A cross-sectional study was conducted between October 2014 to May 2015, at the campus of Zhongshan School of Medicine, Sun Yat-sen University (SYSU), Guangzhou, South China. All the volunteers from 10 to 76 years old included middle school students, undergraduates, teachers, salesclerks and retirees. The pertinent demographic, medical information and potential factors that are related to $S$. aureus nasal carriage and transmission were collected through a standardized questionnaire. Eleven unqualified surveys whose responses were incomplete were eliminating, 295 nasal swabs were sampled. All volunteers, parents, or guardians signed informed consent documents approving the use of their samples for research purposes, and the study was approved by the Ethics Committee of Sun Yat-Sen Memorial Hospital. [Ethical Approval Number:【2017】伦备第 (01) 号].

\section{Bacterial strains}

Both anterior nares were swabbed by rotating a sterile dry cotton swab 5 times inside the nostril. The samples were immediately stored in Copan eSwab Liquid Amies preservation medium (eSwab Collection and Preservation System, Copan Italia, Brescia, Italy). All swabs were kept at $4{ }^{\circ} \mathrm{C}$, transported at room temperature to the department of bacteriology and processed in $4 \mathrm{~h}$. The swabs were streaked on blood agar plates at $35{ }^{\circ} \mathrm{C}$ for $24 \mathrm{~h}$. Gram-positive, $\beta$-hemolytic and coagulase positive isolates were confirmed as $S$. aureus using a Vitek ${ }^{\circledR}$ 2 microbial identification system (bioMérieux, Marcy l' Etoile, France) according to the manufacturer's instructions. All S. aureus were then cultured on MRSA select chromogenic agar. Presumptive MRSA strains, which grew as green colonies on the chromogenic medium, were confirmed by their resistance to cefoxitin and polymerase chain reaction (PCR) for the mecA gene [14].

\section{Antibiotic susceptibility testing}

All $S$. aureus isolates were tested for their susceptibility to the following antibiotics: penicillin, erythromycin, clindamycin, cefuroxime, ceftriaxone, cefotaxime, cefoxitin, gentamicin, rifampicin, imipenem, quinupristin/dalfopristin, tetracycline, teicoplanin, vancomycin, trimethoprim/sulfamethoxazole, ciprofloxacin, and levofloxacin. The sensitivity patterns of methicillin-susceptible $S$. aureus (MSSA) and MRSA strains were determined by disk diffusion method according to 25rd informational supplement (M100-S25) which recommended by the Clinical and Laboratory Standards Institute (CLSI; http:// clsi.org). The inducible clindamycin resistance was determined by D-test. All disks were obtained from Oxoid Ltd (Oxoid, Basingstoke, England). American type culture collection (ATCC) 25923 S. aureus was used as the quality control strain.

\section{Staphylococcal toxin genes detection}

The Panton-Valentine leukocidin $(p v l)$ and the staphylococcal enterotoxin A (sea) and enterotoxin B (seb) genes were detected by PCR as previously described $[14,15]$ (Fig. 1). 


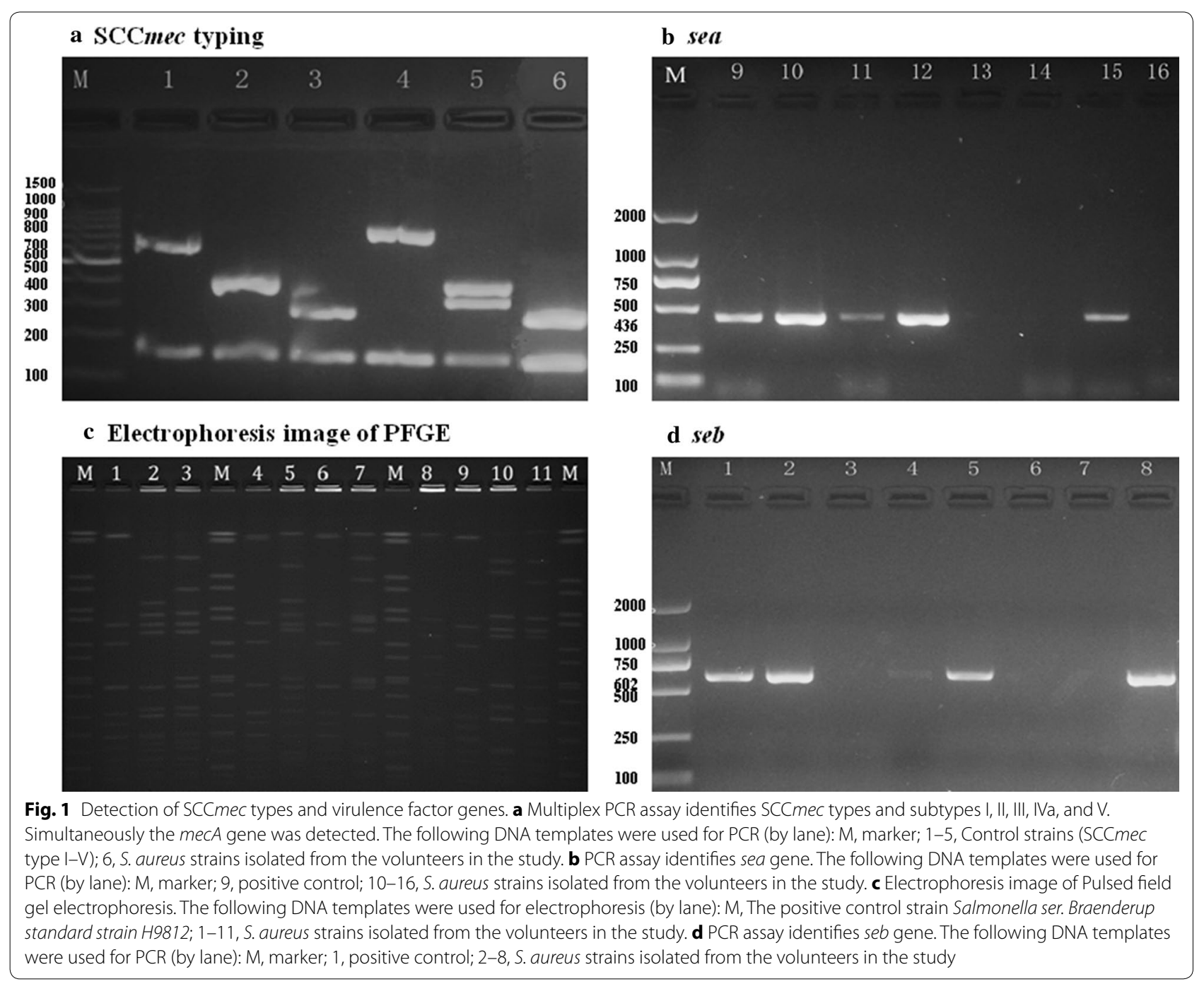

\section{Staphyloccoccal cassette chromosome mec (SCCmec) typing}

Multiplex PCR was performed for SCCmec typing of MRSA isolates by using eight unique pairs of primers for SCCmec types and subtypes I, II, III, IVa, IVb, IVc, IVd, and V, as described previously [16] (Fig. 1). Positive control strains for SCCmec types I (NCTC 10442), II (N315), III (85/2082), and IVa (JCSC 4744), were kindly provided by Dr. Fangyou Yu of the Department of Laboratory Medicine, the First Affiliated Hospital of Wenzhou Medical College.

\section{Multilocus sequence typing (MLST)}

MLST was performed using previously described primers and conditions [17], the sequence types (STs) was obtained through the website http://saureus.mlst.net and the clonal complexes (CCs) were defined by analyzing cluster related STs with the eBURST software (http:// eburst.mlst.net/v3/enter_data/single). A neighbor-joining tree was constructed from the sequence data using MEGA version 6.06 [11]. STs that grouped together with $\geq 70 \%$ bootstrap support were considered part of the same CC.

\section{Pulsed-field gel electrophoresis (PFGE)}

PFGE was performed using SmaI as described previously [18] (Fig. 3). The relatedness of the strains were determined according to the criteria of Tenover et al. [19]. The isolates with $>75 \%$ similarity were clustered in patterns. The dendrograms were generated by analyzing the electrophoretogram with BioNumerics version 5.01 statistical software, according to a simple matching coefficient and the unweighted pair group method with the arithmetic mean (UPGMA) algorithm. The same PFGE patterns 
were grouped as a pulsotype and assigned alphabetically (A, B, C etc.).

\section{Statistical analysis}

Frequencies were obtained and proportions were calculated for categorical variables. The only continuous variable, age, was transformed into a categorical variable using the quartiles of the frequency distribution $(\leq 20,>20-30$, $>30-50,>50$ years). Categorical variables were compared using the Chi square test or the Fisher exact test. Odds ratios (OR), 95\% confidence intervals (CI), and $P$ values were calculated. A $P$ value of $\leq 0.05$ was considered statistically significant. Univariable logistic regression models were applied to determine independent risk factors. Multiple logistic regression analysis was carried out by stepwise backward selection of variables with biological plausibility and a significance level $<0.10$ for entry into the model. Statistical comparisons were performed with SPSS (PASW Statistics 18) software (IBM, Armonk, NY).
All susceptibility data were analyzed using WHONET software, version 5.6.

\section{Results}

Nasal colonization with S. aureus

A total of 295 volunteers were enrolled onto this study. The median age of the participating volunteers was 30.0 years (range $10-76$ years), and $45.8 \%$ (135/295) were male. Distributions of $S$. aureus carriers and noncarriers stratified by population characteristics and variables associated with $S$. aureus carriage in the univariate analysis were shown in Table 1 . The overall prevalence of $S$. aureus carriage was $24.7 \%$ (73/295). The nasal carriage of $S$. aureus was $32.6 \%(44 / 135)$ in males, which is higher than $18.1 \%(29 / 160)$ in females (OR 2.04, 95\% CI 1.27-3.79). The difference between nasal carriage in male and female was statistically significant $(P<0.05)$. Highest nasal carriage, 33.1\% (41/124) (OR 3.30; 95\% CI $1.12-9.75)$ of $S$. aureus was recorded in the age group of

Table 1 Univariate and multivariate analysis of risk factors associated with S. aureus nasal carriage among 295 volunteers at the campus of Zhongshan School of Medicine, Sun Yat-sen University, Guangzhou

\begin{tabular}{|c|c|c|c|c|c|c|}
\hline \multirow[t]{3}{*}{ Characteristic } & \multicolumn{6}{|c|}{ The healthy people $(n=295), n(\%)$} \\
\hline & \multirow{2}{*}{$\begin{array}{l}\text { Carriers }(\mathrm{n}=73) \\
\mathrm{n}(\%)\end{array}$} & \multirow{2}{*}{$\begin{array}{l}\text { Non-carriers }(n=222) \\
n(\%)\end{array}$} & \multicolumn{2}{|c|}{ Univariate } & \multicolumn{2}{|c|}{ Multivariate logistic } \\
\hline & & & $P$ value & OR $(95 \% \mathrm{Cl})$ & $P$ value & OR $(95 \% \mathrm{Cl})$ \\
\hline \multicolumn{7}{|l|}{ Sex } \\
\hline Male & $44(32.6)$ & $91(67.4)$ & 0.005 & $2.04(1.27-3.79)$ & 0.021 & $2.51(1.29-3.91)$ \\
\hline Female & $29(18.1)$ & $131(81.9)$ & & & & \\
\hline \multicolumn{7}{|l|}{ Age, years } \\
\hline$\leq 20$ & $41(33.1)$ & $8(66.9)$ & $<0.001$ & $0.25(0.11-0.54)$ & 0.01 & $3.30(1.12-9.75)$ \\
\hline$>20-30$ & $18(26.5)$ & $50(73.5)$ & 0.021 & $0.31(0.11-0.84)$ & 0.041 & $2.71(1.15-7.51)$ \\
\hline$\geq 30-50$ & $8(17.0)$ & $39(83.0)$ & 0.312 & $0.56(0.18-1.74)$ & 0.451 & $1.91(0.44-4.91)$ \\
\hline$\geq 50$ & $6(10.7)$ & $50(89.3)$ & & 1 & & 1 \\
\hline \multicolumn{7}{|c|}{ Antibiotic use in past 1 month } \\
\hline Yes & $4(20.0)$ & $16(80.0)$ & 0.612 & $0.75(0.24-2.31)$ & & \\
\hline No & $69(25.1)$ & $206(74.9)$ & & & & \\
\hline \multicolumn{7}{|c|}{ Regular contact nasal cavity cleaning } \\
\hline Yes & $13(13.0)$ & $87(87.0)$ & 0.001 & $0.34(0.17-0.65)$ & $<0.001$ & $0.29(0.15-0.56)$ \\
\hline No & $59(30.4)$ & $135(69.6)$ & & & & \\
\hline \multicolumn{7}{|c|}{ Hospitalization in past one year } \\
\hline Yes & $4(36.4)$ & $7(63.6)$ & 0.369 & $1.78(0.51-6.27)$ & & \\
\hline No & $69(24.3)$ & $215(75.7)$ & & & & \\
\hline \multicolumn{7}{|c|}{ Underlying disease } \\
\hline Yes & $11(36.7)$ & $19(63.3)$ & 0.891 & $1.05(0.50-2.21)$ & & \\
\hline No & $62(23.4)$ & $203(76.6)$ & & & & \\
\hline \multicolumn{7}{|c|}{ Household member working in Medical Institutions } \\
\hline Yes & $14(26.4)$ & $39(73.6)$ & 0.689 & $1.15(0.58-2.27)$ & & \\
\hline No & $59(24.4)$ & $183(75.6)$ & & & & \\
\hline
\end{tabular}

Underlying disease: hypertension, diabetes, chronic rhinitis, urticaria, hyperthyroidism

$O R$ odds ratio, $\mathrm{Cl}$ confidence interval 
$\leq 20$ years, followed by $26.5 \%$ (18/68) (OR 2.71, 95\% CI $1.15-7.51), 17.0 \%$ (8/47) (OR 1.91, 95\% CI 0.44-4.91), and $10.7 \%(6 / 56)$ in the age groups of $20-30$ years, $30-50$ years and $>50$ years. There was statistical significant difference $(P<0.05)$ between ages $<20$ years and $20-30$ years. The corresponding rates were $13.0 \%$ $(13 / 100)$ and $30.4 \%$ (60/194) (OR 0.34, 95\% CI 0.17-0.65) between those who clean their nasal frequently or occasionally, respectively, and there was statistical significant difference $(P<0.05)$. In multiple logistic regression analysis, nasal carriage of $S$. aureus was also significantly associated with male, age $\leq 20$ years, and regular cleaning of the nasal cavity.

\section{Antibiotic susceptibility}

Susceptibility pattern of $S$. aureus to various antibiotics is shown in Fig. 2. Among the $73 \mathrm{~S}$. aureus isolates, $61(83.6 \%)$ were resistant to penicillin and $32(43.8 \%)$ to erythromycin. The isolate resistant to tetracycline and clindamycin was found in $13(17.8 \%)$ and $10(13.7 \%)$ isolates respectively. Rates of resistance to cefuroxime, ceftriaxone, cefotaxime, cefoxitin, trimethoprim/sulfamethoxazole, gentamicin, rifampicin, and imipenem were $<10 \%$. All isolates were susceptible to levofloxacin, quinupristin/dalfopristin, teicoplanin, and vancomycin. Among $73 \mathrm{~S}$. aureus isolates, only one isolate $(1.4 \%$, 1/73) was resistant to cefoxitin and further confirmed to be MRSA detecting mecA gene by PCR screening. It was resistant to penicillin, cefuroxime, ceftriaxone, cefotaxime, erythromycin, clindamycin, tetracycline, and gentamycin. The isolate was separated from a 17-year-old female middle school student, who had be in hospital for some days and taken antithyroid drugs because of thyroid problem in past half a year.

\section{Detection of toxin genes and $\mathrm{SCCmec}$ typing}

Detection of toxin genes and $\mathrm{SCCmec}$ typing were shown in Fig. 1. Only one isolate was clearly typed harbored SCCmec III. Among all the S. aureus isolates, 56.5\% detected both sea and seb, 26.1\% detected only sea and $13.0 \%$ detected only seb. And the MRSA isolate was only detected seb. Seven isolates that detected both sea and $s e b$ were found separated from the students who came from the same class. There was not statistical significant difference $(P>0.05)$ among age, gender and profession in detection of enterotoxin gene. Interestingly, the $p v l$ gene was not detected among all the S. aureus isolates in this study.

\section{MLST and PFGE typing}

Twenty-three $S$. aureus isolates with similar drug-resistant spectrum were revealed 12 different sequence types. Among these, ST59, ST188 and ST1 were the most prevalent, accounting for $17.4 \%(4 / 23), 13.0 \%(3 / 23)$ and

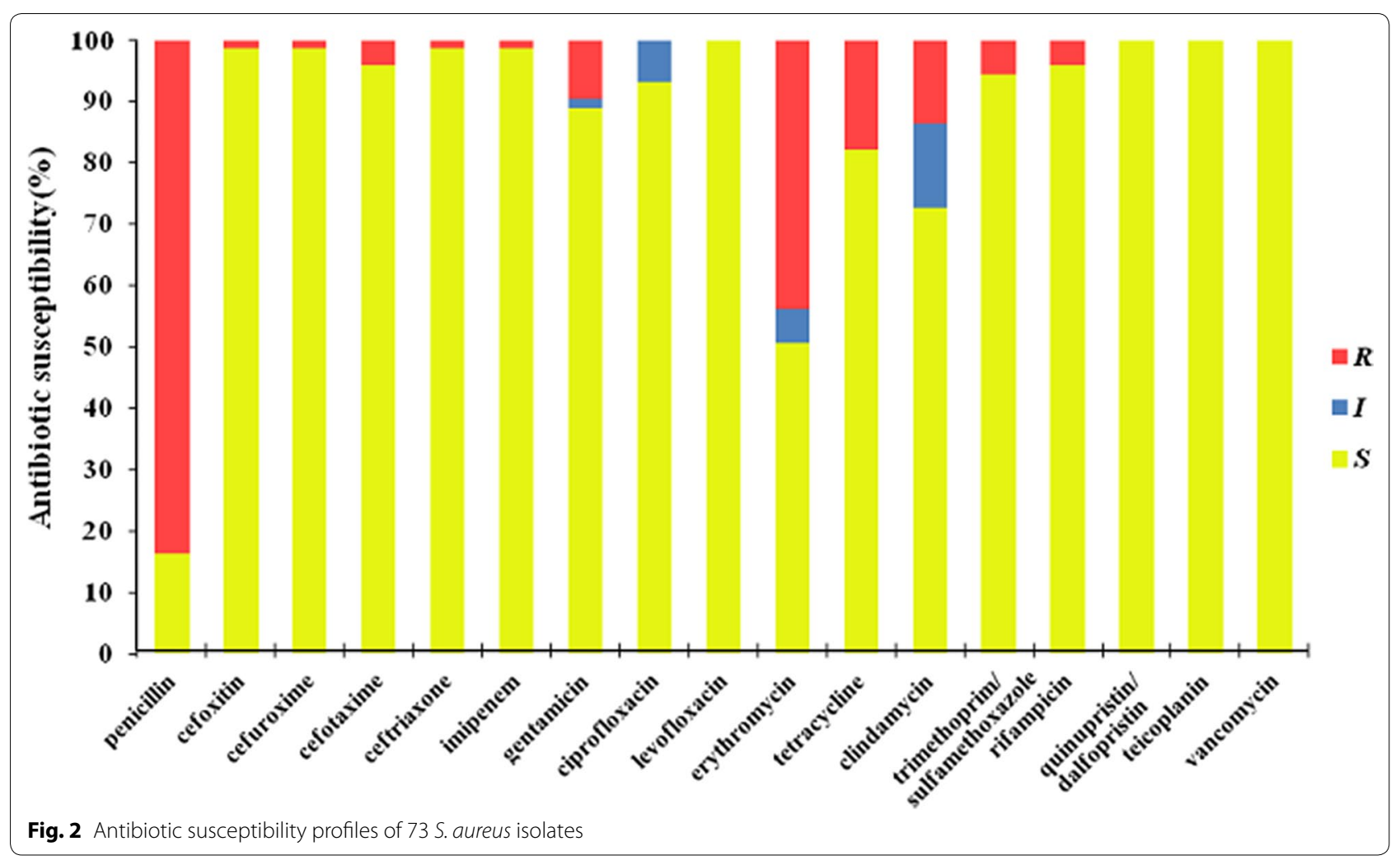


13.0\% (3/23), respectively. Other ST types were ST965, ST30, ST6, ST7, ST5, ST8, ST72, ST537 and ST944. With the eBURST software, $10 \mathrm{CCs}$ were identified. The main CC was CC59 ( $n=5)$, followed by CC188 $(n=3)$, CC1 $(\mathrm{n}=3), \operatorname{CC} 5(\mathrm{n}=3)$, CC6 $(\mathrm{n}=2), \operatorname{CC} 7(\mathrm{n}=2), \operatorname{CC} 30$ $(\mathrm{n}=2), \mathrm{CC} 8(\mathrm{n}=1), \mathrm{CC} 72(\mathrm{n}=1)$ and CC182 $(\mathrm{n}=1)$. The MRSA isolate was revealed ST8 (Table 2). Of the 73 S. aureus isolates collected, 71 isolates consisted of 13 different pulsotypes, while two isolates were untypable. Patterns were classified from A-M, each defining a clone in according with the previously reported interpretive criteria [19]. The most prevalent profiles were A (50.7\%, $36 / 71)$ and $\mathrm{L}(18.3 \%, 13 / 71)$, the MRSA isolate belonged to pulsotype C. $69.4 \%$ (25/36) of the isolates separated from students were came from the same class in profiles A, and $84.6 \%(11 / 13)$ in profile L (Fig. 3).

\section{Discussion}

Staphylococcus aureus nasal carriage is not infrequent in China but few reports on the prevalence and the risk factors of $S$. aureus nasal carriage are found. The findings of this study are of significance to understand $S$. aureus nasal colonization dynamics within the special community, and to design strategies to prevent $S$. aureus infection and dissemination. S. aureus nasal carriage is a global phenomenon among healthy population. But detection rate of $S$. aureus nasal carriage is different in different area. The overall prevalence of $S$. aureus carriage was $24.7 \%$ in this study. Only one $S$. aureus was founded as MRSA (1.4\%). Compared with the general population, this coincides with the recorded prevalence among 2448 healthy people from Beijing and Harbin in Northern China (16.5\%), of which $0.3 \%$ were MRSA and in adults in community settings in Taiwan $(22.1 \%)$ [20, 21]. Besides, previous studies revealed a similar nasal carriage rate (15.4-23.1\%) and low prevalence of MRSA colonization (3.0-9.4\%) in Chinese medical students from different regions. Another study also revealed a similar nasal carriage rate $(20 \%)$ with no MRSA strains identified in military volunteers from Beijing [22]. In addition, it also coincides to multiple reports of CA-MRSA infections on college and high school campuses, with a concentration of cases occurring among student athletes [23]. But, in contrast, the nasal carriage rate of MRSA colonization

Table 2 Demographic characteristics and molecular features of 23 cases with S. aureus carried

\begin{tabular}{|c|c|c|c|c|c|c|c|c|c|}
\hline Case & Age & Gender & Profession & Resistance profile & $p v l$ & sea/seb & ST & $\mathrm{CC}$ & PFGE \\
\hline 1 & 20 & Female & $\mathrm{SCH}$ & PEN, GEN, ERY, TCY, CLI, SXT & - & $+/+$ & 59 & 59 & G \\
\hline 2 & 23 & Male & $\mathrm{SCH}$ & PEN & - & \pm & 6 & 6 & A \\
\hline 3 & 22 & Male & $\mathrm{SCH}$ & PEN, ERY, TCY, CLI & - & \pm & 59 & 59 & G \\
\hline 4 & 21 & Male & $\mathrm{SCH}$ & NR. & - & $-/+$ & 188 & 188 & $A$ \\
\hline 5 & 20 & Female & $\mathrm{SCH}$ & PEN, CTX & - & $+/+$ & 965 & 5 & A \\
\hline 6 & 62 & Female & $\mathrm{SCH}$ & PEN, ERY & - & $+/+$ & 30 & 30 & C \\
\hline 7 & 23 & Female & SOC & PEN & - & $+/+$ & 72 & 72 & B \\
\hline 8 & 70 & Female & SOC & PEN, CIP, CLI & - & $+/+$ & 1 & 1 & A \\
\hline 9 & 17 & Female & SOC & PEN, FOX, CXM, CTX, CRO, IPM, CIP,GEN, ERY, TCY, CLI & - & $-/+$ & 8 & 8 & C \\
\hline 10 & 65 & Female & SOC & PEN & - & $-/+$ & 188 & 188 & A \\
\hline 11 & 62 & Female & SOC & PEN & - & $-/-$ & 188 & 188 & A \\
\hline 12 & 53 & Male & $\mathrm{SCH}$ & PEN, CLI & - & $+/+$ & 1 & 1 & $A$ \\
\hline 13 & 24 & Female & $\mathrm{SCH}$ & PEN, CLI & - & \pm & 1 & 1 & A \\
\hline 14 & 13 & Female & $\mathrm{SCH}$ & GEN, ERY, CLI, SXT & - & $+/+$ & 5 & 5 & A \\
\hline 15 & 14 & Male & $\mathrm{SCH}$ & ERY & - & \pm & 6 & 6 & A \\
\hline 16 & 14 & Male & $\mathrm{SCH}$ & PEN, GEN, ERY & - & \pm & 30 & 30 & C \\
\hline 17 & 14 & Female & $\mathrm{SCH}$ & PEN, ERY, TCY, CLI & - & \pm & 59 & 59 & $\mathrm{H}$ \\
\hline 18 & 14 & Male & $\mathrm{SCH}$ & PEN, GEN, ERY, TCY & - & $+/+$ & 965 & 5 & A \\
\hline 19 & 13 & Female & $\mathrm{SCH}$ & PEN, GEN, ERY, CLI, SXT & - & $+/+$ & 7 & 7 & K \\
\hline 20 & 14 & Male & $\mathrm{SCH}$ & NR. & - & $+/+$ & 59 & 59 & G \\
\hline 21 & 13 & Female & $\mathrm{SCH}$ & ERY & - & $+/+$ & 944 & 182 & E \\
\hline 22 & 14 & Male & $\mathrm{SCH}$ & NR. & - & $+/+$ & 7 & 7 & A \\
\hline 23 & 14 & Male & $\mathrm{SCH}$ & NR. & - & $+/+$ & 537 & 59 & G \\
\hline
\end{tabular}

$\mathrm{SCH}$ the volunteers who work or study in the college, $\mathrm{SOC}$ the volunteers who work beside the college, $p v /$ Panton-Valentine leukocidin gene, sea staphylococcal enterotoxin A gene, seb staphylococcal enterotoxin B gene, ST sequence type, CC clonal complexe, PFGE pulsed-field gelelectrophoresis, $P E N$ penicillin, FOX cefoxitin, CXM cefuroxime, CTX cefotaxime, $C R O$ ceftriaxone, IPM imipenem, $T C Y$ tetracycline, CIP ciprofloxacin, $C L I$ clindamycin, ERY erythromycin, GEN gentamicin, SXT trimethoprim/sulfamethoxazole, $N R$. the isolate is sensitive to all the antibiotics, - negative, + positive 


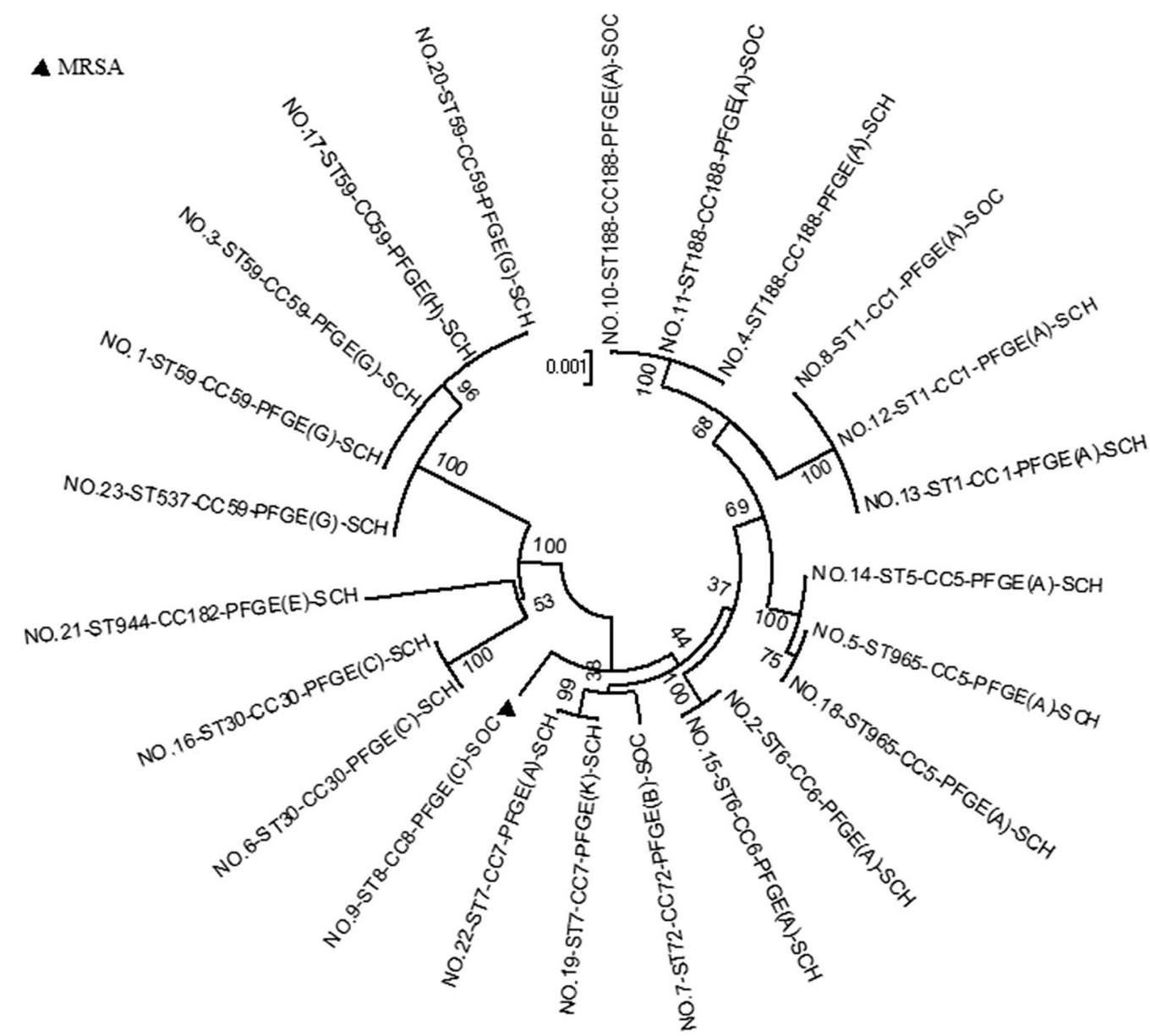

Fig. 3 A neighbour-joining tree reveals phylogenetic relationships of $23 \mathrm{~S}$. aureus strains isolated from the volunteers in the study. The neighbor joining tree was based on the concatenated sequences of each of the 23 sequence types noted in the combined dataset, as determined using the S. aureus MLST database (http://saureus.mlst.net/) and implemented in MEGA v6.06 using Kimura-2-parameter distances. The relationships shown were based on 1000 re-samplings for bootstrapping. Each clonal complex (CC) is composed of STs that cluster with a $\geq 70 \%$ bootstrap confidence value. Bsides, Bayesian phylogram indicating the evolutionary relationships of $S$. aureus strains analyzed in this study. PFGE, pulsed-field gel electrophoresis; $\mathrm{SCH}$, the volunteers who work or study in the college; SOC, the volunteers who work beside the college

was found to be $11.6 \%$ in a cohort of healthy children aged $\leq 14$ years in community settings in Taiwan over a 5-year period [24], which was higher than that in this study. Compared with other nations, the detection rate of S. aureus nasal carriage is lower than that in America and Europe (20-30\%) [25]. which coincides to the report that the detection rate of MRSA among adults is $1 \%$ in Thailand [26], 0.2\% in Northern Europe and 3\% in Northern America [27], but is lower than $8.6 \%$ in Mexico [28]. In addition, the analogous report that the detection rate of S. aureus at Wenzhou Medical College in Wenzhou was $15.4 \%$, of which $3.0 \%$ were MRSA [29]. Besides, the study from Brasil reported that in a medical student community, the detection rate of $S$. aureus and MRSA were 20.6 and 3.4\% [30]. In contrast, the detection rate of S. aureus in this study is higher than the analogous report, but the detection rate of MRSA is lower. In conclusion, though the special community living or working in crowded conditions are more likely to expose to $S$. aureus colonization and infection [29], but the nasal carriage detection rate of MRSA is not higher.

Previous studies have found that young age, male sex, chronic sinusitis, nonuse of antibiotics, the length of hospital stay, less education, and drug use were the risk factors associated with $S$. aureus colonization [31, 32]. Yan et al. [20] found that the populations that typically lived under crowded conditions would have higher opportunities for transmission and non-Han Chinese, youth male and chronic disease were the most possibly risk factors of nasal S. aureus carriage in healthy population. Higher S. aureus carriage rates were also found in men, individuals with obesity and children in Chinese medical college 
campus [29]. Another study reported that insufficient immunity, crowds or closed contacts, and inactivity was an ideal setting for S. aureus [22]. In Taiwan, a study also found that crowded environments, such as living with a greater number of children and attending day care, significantly increased the risk of MRSA colonization [24]. Our study also found that higher $S$. aureus carriage rates were associated with being male, young age and nonstandard nasal cleaning habits. Previous studies have reported that predisposes healthy individuals and transplant recipients to $S$. aureus nasal carriage with $S$. aureus will be affected by the human leukocyte antigen (HLA) DR3 antigen [13, 33]. Thus, gender specific frequencies of HLA haplotypes may lead to differential susceptibilities between male and female. However, HLA DR3 haplotype frequencies among the carriers have not been investigated. Cleaning the nasal cavity with regularity can protect against nasal colonization by S. aureus [34]. According to the standardized questionnaire we found that female volunteers were more likely to clean the nasal cavities with regularity, so it may be an important reason why the nasal carriage of $S$. aureus in males was higher than in females. So it was absolutely essential to clean the nasal cavity with regularity among those who were exposed to higher $S$. aureus carriage rates. Unfortunately, we could not find any difference between nasal carriage in the healthy people and the population under chronic disease was statistically significant because the number of the population under chronic disease was too small. It was observed that pneumococcal competition at the Youth of life would lead to a negative correlation for the cocolonization of S. aureus and Streptococcus pneumonia [35]. Besides, the volunteers whose ages $\leq 20$ years were more likely to study together for more than $7 \mathrm{~h}$ per day, so they had more closely intimate contact with each other. What's more, compared with adults, they would not clean the nasal cavity with regularity. So it may be the explanation for the phenomenon that higher S. aureus carriage rates were associated with young age.

As known, the object of study to MLST was always MRSA but not MSSA. CC5, CC8, CC188, ST398 and CC59 $[20,21,36,37]$ were the major CCs among the $S$. aureus strains separated from both nasal and clinical according to those previous studies from China. But ST7 and CC188 occurred quite a lot in the recent Chinese studies, which indicated that the majority of the MSSA clones observed in China are globally distributed [36, 38]. In this study, $23 \mathrm{~S}$. aureus isolates were separated into 12 STs, which belonged to $10 \mathrm{CCs}$, including 4 major CCs: CC59, CC188, CC1 and CC5, respectively. This coincides with the study to MLST of the $S$. aureus strains separated from nasal among healthy adults in China [20]. And ST59, which belonged to CC59, was the major ST of
CA-MRSA in China [36], what's more, the major type of CA-MRSA in Taiwan was ST59-SCCmecV (pvl-positive), followed by ST59-SCCmecIV (pvl-negative), but 46-59\% MRSA isolates that separated from the nasal of healthy adults belonged to ST59-SCCmecIV (pvl-negative). Interestingly, the MRSA isolate detected in this study belonged to ST8-SCCmecIII ( $p v l$-negative), which was the endemic genotype of hospital-acquired MRSA (HAMRSA) but not CA-MRSA in China [39]. This finding lends further support to the notion that HA-MRSA was going to instead of CA-MRSA by nasal carriage transmission because the parasitifer of the MRSA isolates had a history of exposure to a hospital environment.

PFGE electrophoresis could separate large fragments of DNA, so it was recommended as the standard technique for $S$. aureus genotyping, using for pathogen traceability research and the investigation of hospital infection outbreak [40]. In this study, the $S$. aureus isolates separated from the population in the same class had the same PFGE pulsotype, which indicated that the $S$. aureus may spread from carrier to non carrier by nasal.

Panton-Valentine leukocidin [41, 42], encoded by $p v l$ gene, was a toxin that lyses leukocytes in a receptordependent fashion and associated with skin and soft tissue infection. Previous study showed that the acquisition of a mobile genetic element carrying the genes coding for $p v l$ gene was mainly based on the finding that most initially found CA-MRSA clones $p v l$-encoding genes, while HA-MRSA commonly do not [41]. But the role of $p v l$ gene in the pathogenesis of CA-MRSA infections, in particular skin infection as the most common manifestation of CA-MRSA disease, is still controversial [43]. Interestingly, the $p v l$ gene was not detected among all the S. aureus isolates in this study. It coincidesed with the study by Sanchini et al. [44], who collected 18 CA-MRSA strains from all over Italy but only found one of these tested positive for $p v l$ gene.

Staphylococcus aureus enterotoxins (SE) are major causes of staphylococcal food poisoning [45]. Staphylococcal enterotoxin A and enterotoxin B genes, which were associated with severe disease such as necrotizing soft tissue infections, were confirmed as the most abundant toxin genes in clinical S. aureus isolates from patients and children in China [46, 47]. Previous reports have found that isolates from patients with bacteremia always detected sea and seb was identified in isolates from sputum samples, and the most abundant toxin genes in clinical $S$. aureus isolates from patients and children in China were sea and seb [46, 47]. Our recent study have shown that 56.5\% CA-MRSA and 61.5\% HAMRSA carried the sea gene. However, seb gene was only detected in CA-MRSA isolates (52.2\%) [48]. Another previous investigation has demonstrated that a significantly 
higher proportion of sea gene-positive isolates came from the community residents compared with the healthcare workers (26.1 vs. $5.6 \%, P=0.024)$ [34]. The important finding of the current study was the high rate of detection of the enterotoxin genes in S. aureus (56.5\% detected both sea and seb), which indicated that $S$. aureus carriers are at risk of autoinfection.

Broader concerns should be paid on the antimicrobial resistance problems in S. aureus. S. aureus strains can differ in their susceptibility profiles $[49,50]$. In this study, we found that there was a high rate of resistance against penicillin and erythromycin. The reason might be the excessive use of penicillin and macrolides [49, 50]. Fortunately, most of the isolates remained sensitive to the majority of antibiotics, gentamicin, rifampicin, trimethoprim/sulfamethoxazole, quinupristin/dalfopristin and teicoplanin. Therefore, formulating more strategies for rational use of antibiotics are urgently required.

There are limitations to note about the current work. Most importantly, as a cross-sectional study, to detect variations in colonization patterns, e.g. persistent carriers, intermittent carriers, or non-carriers was unpractical. Secondly, because the number of volunteers is limit, recruitment of subjects from the college may mean that the results are not generalizable to the population of the medicos in Guangzhou as a whole, despite the good cross sections of age and occupation among the test subjects. Thirdly, sampling only the nostrils without including other body parts may underestimate the frequency of MRSA carriage overall [50].

\section{Conclusions}

This study showed that being male, young age (2030 years) and irregularity nasal cleaning are more likely to be colonized by $S$. aureus. The finding lends further evidence of molecular heterogeneity among $S$. aureus isolates from the populations in the medical college in Guangzhou.

\section{Authors' contributions}

YDY, SYH and BJC contributed to the design of the study and the writing of the manuscript. XYX, LJN and XLD performed the experiments. YL, XQW and HYL assisted with quantitative data collection and volunteers follow up. All authors read and approved the final manuscript.

\section{Author details}

1 Department of Laboratory, Guangdong Provincial Key Laboratory of Malignant Tumor Epigenetics and Gene Regulation, Sun Yat-Sen Memorial Hospital, Sun Yat-Sen University, Guangzhou 510120, China. ${ }^{2}$ Cross Infection Control Office, Sun Yat-Sen Memorial Hospital, Sun Yat-Sen University, Guangzhou 510120, Guangdong, China. ${ }^{3}$ Breast Tumor Center, Sun Yat-Sen Memorial Hospital, Sun Yat-Sen University, Guangzhou 510120, Guangdong, China.

\section{Acknowledgements}

This work was supported by Grant [2013]163 from Key Laboratory of Malignant Tumor Molecular Mechanism and Translational Medicine of Guangzhou Bureau of Science and Information Technology.

\section{Competing interests}

The authors declare that they have no competing interests.

\section{Ethics approval and consent to participate}

The Ethics Committee has reviewed the project entitled" Factors associated with Staphylococcus aureus nasal carriage and molecular characteristics among the general population at a Medical College Campus in Guangzhou, South China". This project is proposed by your center and in accordance with the ethics review regulations. The Ethics Committee has approved this project. It must be conducted in conformance with the proposal. [Ethical Approval Number:【2017】伦备第 (01) 号]

\section{Funding}

This work was supported by grants from the National Natural Science Foundation of China (81272897), the Science and Technology Foundation of the Guangdong Province (2014A050503029), and the Sun Yat-sen Initiative Program for Scientific Research (YXQH201701). The funders played no role in the study design, data collection and analysis, decision to publish, or preparation of the manuscript.

\section{Publisher's Note}

Springer Nature remains neutral with regard to jurisdictional claims in publishedmaps and institutional affiliations.

Received: 28 January 2017 Accepted: 6 April 2017

Published online: 11 April 2017

\section{References}

1. Kluytmans J, van Belkum A, Verbrugh H. Nasal carriage of Staphylococcus aureus: epidemiology, underlying mechanisms, and associated risks. Clin Microbiol Rev. 1997;10:505-20.

2. Yu VL, Goetz A, Wagener M, Smith PB, Rihs JD, Hanchett J, Zuravleff JJ. Staphylococcus aureus nasal carriage and infection in patients on hemodialysis: efficacy of antibiotic prophylaxis. N Engl J Med. 1986;315:91-6.

3. Young BC, GolubchikT, Batty EM, Fung R, Larner-Svensson H, Votintseva AA, Miller RR, Godwin H, Knox K, Everitt RG, et al. Evolutionary dynamics of Staphylococcus aureus during progression from carriage to disease. Proc Natl Acad Sci USA. 2012;109:4550-5.

4. Munckhof WJ, Nimmo GR, Schooneveldt JM, Schlebusch S, Stephens AJ, Williams G, Huygens F, Giffard P. Nasal carriage of Staphylococcus aureus, including community-associated methicillin-resistant strains, in Queensland adults. Clin Microbiol Infect. 2009;15:149-55.

5. Salgado CD, Farr BM, Calfee DP. Community-acquired methicillin-resistant Staphylococcus aureus: a meta-analysis of prevalence and risk factors. Clin Infect Dis. 2003;36:131-9.

6. Herold BC, Immergluck LC, Maranan MC, Lauderdale DS, Gaskin RE, BoyleVavra S, Leitch CD, Daum RS. Community-acquired methicillin-resistant Staphylococcus aureus in children with no identified predisposing risk. JAMA. 1998;279:593-8.

7. Centers for Disease Control and Prevention. Four pediatric deaths from community-acquired methicillin-resistant Staphylococcus aureusMinnesota and North Dakota, 1997-1999. MMWR Morb Mortal Wkly Rep. 1999:48:707-10.

8. Wertheim HF, Vos MC, Ott A, van Belkum A, Voss A, Kluytmans JA, van Keulen $\mathrm{PH}$, Vandenbroucke-Grauls CM, Meester MH, Verbrugh HA. Risk and outcome of nosocomial Staphylococcus aureus bacteraemia in nasal carriers versus non-carriers. Lancet. 2004;364:703-5.

9. von Eiff C, Becker K, Machka K, Stammer H, Peters G. Nasal carriage as a source of Staphylococcus aureus bacteremia, Study Group. N Engl J Med. 2001;344:11-6.

10. Enright MC, Robinson DA, Randle G, Feil EJ, Grundmann H, Spratt BG. The evolutionary history of methicillin-resistant Staphylococcus aureus (MRSA) Proc Natl Acad Sci USA. 2002;99:7687-92.

11. Liu Y, Wang H, Du N, Shen E, Chen H, Niu J, Ye H, Chen M. Molecular evidence for spread of two major methicillin-resistant Staphylococcus aureus clones with a unique geographic distribution in Chinese hospitals. Antimicrob Agents Chemother. 2009;53:512-8. 
12. Noble WC. Carriage of Staphylococcus aureus and beta haemolytic streptococci in relation to race. Acta Derm Venereol. 1974;54(5):403-5.

13. Kinsman OS, McKenna R, Noble WC. Association between histocompatability antigens (HLA) and nasal carriage of Staphylococcus aureus. J Med Microbiol. 1983;16:215-20.

14. Larsen AR, Stegger M, Sorum M. spa typing directly from a mecA, spa and pvl multiplex PCR assay-a cost-effective improvement for methicillin-resistant Staphylococcus aureus surveillance. Clin Microbiol Infect. 2008;14:611-4.

15. Fernandes $P$, Ferreira BS, Cabral JM. Solvent tolerance in bacteria: role of efflux pumps and cross-resistance with antibiotics. Int J Antimicrob Agents. 2003;22:211-6.

16. Zhang K, McClure JA, Elsayed S, Louie T, Conly JM. Novel multiplex PCR assay for characterization and concomitant subtyping of staphylococcal cassette chromosome mec types I to V in methicillin-resistant Staphylococcus aureus. J Clin Microbiol. 2005;43:5026-33.

17. Enright MC, Day NP, Davies CE, Peacock SJ, Spratt BG. Multilocus sequence typing for characterization of methicillin-resistant and methicillin-susceptible clones of Staphylococcus aureus. J Clin Microbiol. 2000;38:1008-15.

18. Ben Slama K, Gharsa H, Klibi N, Jouini A, Lozano C, Gomez-Sanz E, Zarazaga M, Boudabous A, Torres C. Nasal carriage of Staphylococcus aureus in healthy humans with different levels of contact with animals in Tunisia: genetic lineages, methicillin resistance, and virulence factors. Eur J Clin Microbiol Infect Dis. 2010;30:499-508.

19. Tenover FC, Arbeit RD, Goering RV, Mickelsen PA, Murray BE, Persing DH, Swaminathan B. Interpreting chromosomal DNA restriction patterns produced by pulsed-field gel electrophoresis: criteria for bacterial strain typing. J Clin Microbiol. 1995;33:2233-9.

20. Yan X, Song Y, Yu X, Tao X, Yan J, Luo F, Zhang H, Zhang J, Li Q, He L, et al. Factors associated with Staphylococcus aureus nasal carriage among healthy people in Northern China. Clin Microbiol Infect. 2015;21:157-62.

21. Wang JT, Liao CH, Fang CT, Chie WC, Lai MS, Lauderdale TL, Lee WS, Huang JH, Chang SC. Prevalence of and risk factors for colonization by methicillin-resistant Staphylococcus aureus among adults in community settings in Taiwan. J Clin Microbiol. 2009;47:2957-63.

22. Qu F, Cui E, Guo T, Li H, Chen S, Liu L, Han W, Bao C, Mao Y, Tang YW. Nasal colonization of and clonal transmission of methicillin-susceptible Staphylococcus aureus among Chinese military volunteers. J Clin Microbiol. 2009:48:64-9.

23. Cohen PR. The skin in the gym: a comprehensive review of the cutaneous manifestations of community-acquired methicillin-resistant Staphylococcus aureus infection in athletes. Clin Dermatol. 2008;26:16-26.

24. Chen CJ, Hsu KH, Lin TY, Hwang KP, Chen PY, Huang YC. Factors associated with nasal colonization of methicillin-resistant Staphylococcus aureus among healthy children in Taiwan. J Clin Microbiol. 2010:49:131-7.

25. Mainous AG 3rd, Hueston WJ, Everett CJ, Diaz VA. Nasal carriage of Staphylococcus aureus and methicillin-resistant $S$ aureus in the United States, 2001-2002. Ann Fam Med. 2006;4:132-7.

26. Kitti T, Boonyonying K, Sitthisak S. Prevalence of methicillin-resistant Staphylococcus aureus among university students in Thailand. Southeast Asian JTrop Med Public Health. 2012;42:1498-504.

27. Walsh EE, Greene L, Kirshner R. Sustained reduction in methicillin-resistant Staphylococcus aureus wound infections after cardiothoracic surgery. Arch Intern Med. 2010:171:68-73.

28. Hamdan-Partida A, Sainz-Espunes T, Bustos-Martinez J. Characterization and persistence of Staphylococcus aureus strains isolated from the anterior nares and throats of healthy carriers in a Mexican community. J Clin Microbiol. 2010;48:1701-5.

29. Du J, Chen C, Ding B, Tu J, Qin Z, Parsons C, Salgado C, Cai Q, Song Y, Bao $Q$, et al. Molecular characterization and antimicrobial susceptibility of nasal Staphylococcus aureus isolates from a Chinese medical college campus. PLOS ONE. 2011;6:e27328.

30. Gushiken CY, Medeiros LB, Correia BP, Souza JM, Moris DV, Pereira VC, Giuffrida R, Rodrigues MV. Nasal carriage of resistant Staphylococcus aureus in a medical student community. An Acad Bras Cienc. 2016;88:1501-9.

31. Al-Rawahi GN, Schreader AG, Porter SD, Roscoe DL, Gustafson R, Bryce EA. Methicillin-resistant Staphylococcus aureus nasal carriage among injection drug users: six years later. J Clin Microbiol. 2008:46:477-9.

32. Graham PL 3rd, Lin SX, Larson EL. A U.S. population-based survey of Staphylococcus aureus colonization. Ann Intern Med. 2006;144:318-25.
33. Giarola LB, Dos Santos RR, Bedendo J, da Silva Junior WV, Borelli SD. HLA molecules and nasal carriage of Staphylococcus aureus isolated from dialysis and kidney transplant patients at a hospital in Southern Brazil. BMC Res Notes. 2012;5:90.

34. Chen B, Dai X, He B, Pan K, Li H, Liu X, Bao Y, Lao W, Wu X, Yao Y, et al. Differences in Staphylococcus aureus nasal carriage and molecular characteristics among community residents and healthcare workers at Sun Yat-Sen University, Guangzhou, Southern China. BMC Infect Dis. 2015;15:303.

35. Bogaert D, van Belkum A, Sluijter M, Luijendijk A, de Groot R, Rumke HC, Verbrugh HA, Hermans PW. Colonisation by Streptococcus pneumoniae and Staphylococcus aureus in healthy children. Lancet. 2004:363:1871-2.

36. Chen FJ, Huang IW, Wang CH, Chen PC, Wang HY, Lai JF, Shiau YR, Lauderdale TL. mecA-positive Staphylococcus aureus with low-level oxacillin MIC in Taiwan. J Clin Microbiol. 2012;50:1679-83.

37. Zhao C, Liu Y, Zhao M, Yu Y, Chen H, Sun Q, Jiang W, Han $S$, Xu Y, Chen $\mathrm{M}$, et al. Characterization of community acquired Staphylococcus aureus associated with skin and soft tissue infection in Beijing: high prevalence of PVL + ST398. PLOS ONE. 2012:7:e38577.

38. Yu F, Li T, Huang X, Xie J, Xu Y, Tu J, Qin Z, Parsons C, Wang J, Hu L, et al. Virulence gene profiling and molecular characterization of hospitalacquired Staphylococcus aureus isolates associated with bloodstream infection. Diagn Microbiol Infect Dis. 2012;74:363-8.

39. Chen H, Liu Y, Jiang X, Chen M, Wang H. Rapid change of methicillinresistant Staphylococcus aureus clones in a Chinese tertiary care hospital over a 15-year period. Antimicrob Agents Chemother. 2010;54:1842-7.

40. Murchan S, Kaufmann ME, Deplano A, de Ryck R, Struelens M, Zinn CE, Fussing V, Salmenlinna S, Vuopio-Varkila J, El Solh N, et al. Harmonization of pulsed-field gel electrophoresis protocols for epidemiological typing of strains of methicillin-resistant Staphylococcus aureus: a single approach developed by consensus in 10 European laboratories and its application for tracing the spread of related strains. J Clin Microbiol. 2003;41:1574-85.

41. Tristan A, Bes M, Meugnier H, Lina G, Bozdogan B, Courvalin P, Reverdy ME, Enright MC, Vandenesch F, Etienne J. Global distribution of PantonValentine leukocidin-positive methicillin-resistant Staphylococcus aureus, 2006. Emerg Infect Dis. 2007;13:594-600.

42. Spaan AN, Henry T, van Rooijen WJ, Perret M, Badiou C, Aerts PC, Kemmink J, de Haas CJ, van Kessel KP, Vandenesch F, et al. The staphylococcal toxin Panton-Valentine Leukocidin targets human C5a receptors. Cell Host Microbe. 2013;13:584-94.

43. Otto M. Community-associated MRSA: what makes them special? Int J Med Microbiol. 2013;303:324-30.

44. Sanchini A, Campanile F, Monaco M, Cafiso V, Rasigade JP, Laurent F, Etienne J, Stefani S, Pantosti A. DNA microarray-based characterisation of Panton-Valentine leukocidin-positive community-acquired methicillinresistant Staphylococcus aureus from Italy. Eur J Clin Microbiol Infect Dis. 2011:30:1399-408.

45. Argudin MA, Mendoza MC, Rodicio MR. Food poisoning and Staphylococcus aureus enterotoxins. Toxins (Basel). 2010;2:1751-73.

46. He W, Chen H, Zhao C, Zhang F, Li H, Wang Q, Wang X, Wang H. Population structure and characterisation of Staphylococcus aureus from bacteraemia at multiple hospitals in China: association between antimicrobial resistance, toxin genes and genotypes. Int J Antimicrob Agents. 2013:42:211-9.

47. Wu D, Li X, Yang Y, Zheng Y, Wang C, Deng L, Liu L, Li C, Shang Y, Zhao C, et al. Superantigen gene profiles and presence of exfoliative toxin genes in community-acquired meticillin-resistant Staphylococcus aureus isolated from Chinese children. J Med Microbiol. 2010;60:35-45.

48. Xie X, Bao Y, Ouyang N, Dai X, Pan K, Chen B, Deng Y, Wu X, Xu F, Li H, et al. Molecular epidemiology and characteristic of virulence gene of community-acquired and hospital-acquired methicillin-resistant Staphylococcus aureus isolates in Sun Yat-sen Memorial hospital, Guangzhou, Southern China. BMC Infect Dis. 2016;16:339.

49. Mehndiratta PL, Gur R, Saini S, Bhalla P. Staphylococcus aureus phage types and their correlation to antibiotic resistance. Indian J Pathol Microbiol. 2010;53:738-41.

50. Treesirichod A, Hantagool S, Prommalikit O. Nasal carriage and antimicrobial susceptibility of Staphylococcus aureus among medical students at the HRH Princess Maha Chakri Sirindhorn Medical Center, Thailand: a cross sectional study. J Infect Public Health. 2013;6:196-201. 